\title{
Synthesis and thermoelectric properties of $\mathrm{Re}_{3} \mathrm{As}_{6.6} \mathrm{In}_{\mathbf{0 . 4}}$ with $\mathrm{Ir}_{3} \mathrm{Ge}_{\mathbf{7}}$ crystal structure
}

\author{
Valeriy Y. Verchenko ${ }^{1}$, Anton S. Vasiliev ${ }^{1}$, Alexander A. Tsirlin², \\ Vladimir A. Kulbachinskii ${ }^{3}$, Vladimir G. Kytin ${ }^{3}$ and Andrei V. Shevelkov ${ }^{* 1, \S}$
}

\section{Full Research Paper}

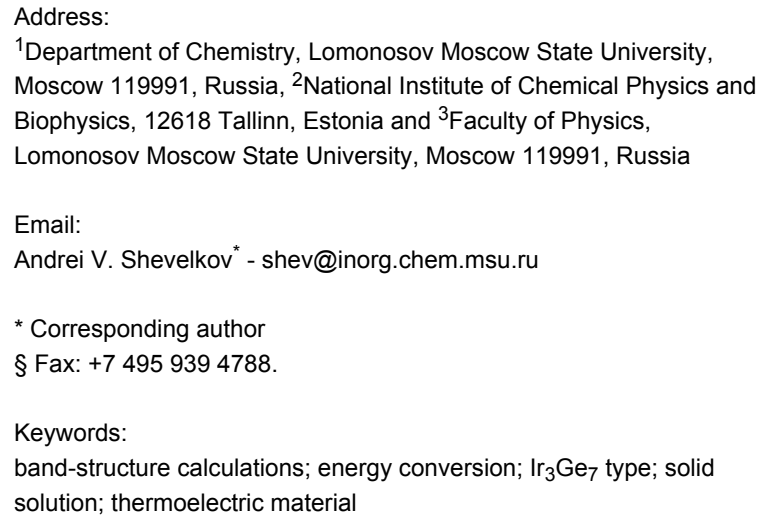

${ }^{1}$ Department of Chemistry, Lomonosov Moscow State University, Moscow 119991, Russia, ${ }^{2}$ National Institute of Chemical Physics and Biophysics, 12618 Tallinn, Estonia and ${ }^{3}$ Faculty of Physics, Lomonosov Moscow State University, Moscow 119991, Russia

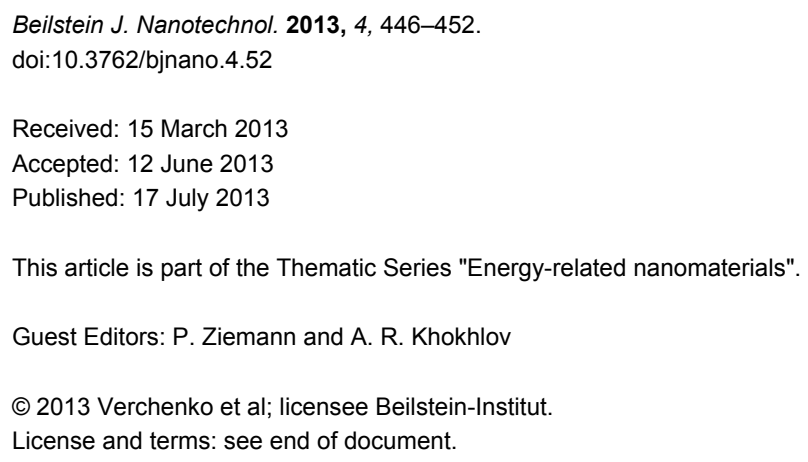

\begin{abstract}
The $\operatorname{Re}_{3} \operatorname{As}_{7-x} \mathrm{In}_{x}$ solid solution was prepared for $x \leq 0.5$ by heating the elements in stoichiometric ratios in evacuated silica tubes at $1073 \mathrm{~K}$. It crystallizes with the $\mathrm{Ir}_{3} \mathrm{Ge}_{7}$ crystal structure, space group $\mathrm{Im}-3 \mathrm{~m}$, with a unit-cell parameter $a$ ranging from 8.716 to $8.747 \AA$. The crystal structure and properties were investigated for a composition with $x=0.4$. It is shown that indium substitutes arsenic exclusively at one crystallographic site, such that the As-As dumbbells with $d_{\text {As- }} \mathrm{As}=2.54 \AA$ remain intact. $\operatorname{Re}_{3} \mathrm{As}_{6.6} \mathrm{In}_{0.4}$ behaves as a bad metal or heavily doped semiconductor, with electrons being the dominant charge carriers. It possesses high values of Seebeck coefficient and low thermal conductivity, but relatively low electrical conductivity, which leads to rather low values of the thermoelectric figure of merit.
\end{abstract}

\section{Introduction}

Thermoelectric materials with good efficiency are highly awaited by modern power engineering. Utilizing either the Seebeck or Peltier effects, it is possible to produce electricity from waste heat (e.g., that stemming from combustion in car engines) or to cool an environment under an external power supply. However, the efficiency of these processes depends on the efficiency of the thermoelectric material in question, which is defined by the value of the figure of merit $Z T=T S^{2} \sigma / \kappa$, where $T$ is the absolute temperature, $S$ the Seebeck coefficient, $\sigma$ the electrical conductivity, and $\kappa$ the thermal conductivity. It is shown in the literature [1] that the best thermoelectric materials are to be sought among narrow-gap semiconductors 
composed of heavy elements, in which structural features favor low thermal conductivity [2]. Attempts to improve the $Z T$ value have led to the investigation of various types of thermoelectrics beyond the long-known lead and bismuth tellurides [3,4] Among new candidates are the filled skutterudites $[5,6]$, semiconducting clathrates [7], disordered materials such as $\mathrm{Zn}_{4} \mathrm{Sb}_{3}$ [8], and various inorganic and intermetallic compounds with complex crystal structures $[9,10]$. Compounds with the $\mathrm{Ir}_{3} \mathrm{Ge}_{7}$ structure type, namely $\mathrm{Mo}_{3} \mathrm{Sb}_{5+\delta} \mathrm{Te}_{2-\delta}[11], \mathrm{Nb}_{3} \mathrm{Sb}_{2} \mathrm{Te}_{5}$ [12] and $\mathrm{Re}_{3} \mathrm{As}_{7-x} \mathrm{Ge}_{x}$ [13], belong to the latter type of potential thermoelectric materials and have recently shown promising $Z T$ values.

All members of the $\mathrm{Ir}_{3} \mathrm{Ge}_{7}$ family crystallize in the cubic space group $\mathrm{Im}-3 m$ and feature the $\mathrm{M}-\mathrm{M}$ dumbells inside the Archimedean antiprism of the non-transition-element atoms. The strong hybridization of the transition-metal d-orbitals with the p-orbitals of a non-transition element may lead to the opening of a band gap near the Fermi level [14]. As a result, compounds with 55 valence electrons per formula unit (f.u.) exhibit semiconductor-like behavior. The number of valence electrons can be tuned through the solid-solution formation. For instance, $\mathrm{Mo}_{3} \mathrm{Sb}_{5} \mathrm{Te}_{2}$ and $\mathrm{Re}_{3} \mathrm{As}_{6} \mathrm{Ge}$ adopt $55 e^{-}$per f.u. and should be semiconductors according to the band structure calculations. Their doped analogues, $\mathrm{Mo}_{3} \mathrm{Sb}_{5.4} \mathrm{Te}_{1.6}$ and $\mathrm{Re}_{3} \mathrm{As}_{6.4} \mathrm{Ge}_{0.6}$, display minor deviation from $55 e^{-}$per formula. They behave as heavily doped semiconductors and possess the $Z T$ values of 0.8 at $1050 \mathrm{~K}$ and 0.3 at $700 \mathrm{~K}$, respectively [13] To expand the $\mathrm{Ir}_{3} \mathrm{Ge}_{7}$ family and search for new promising thermoelectrics, we investigated different ways of obtaining new solid solutions based on $\mathrm{Re}_{3} \mathrm{As}_{7}$ or $\mathrm{Mo}_{3} \mathrm{Sb}_{7}$ compounds. In this paper, we report on the synthesis of the $\mathrm{Re}_{3} \mathrm{As}_{7-x} \mathrm{In}_{x}$ solid solution $(x \leq 0.5)$, its crystal and electronic structures, and its thermoelectric properties.

\section{Experimental \\ Synthesis and analysis}

Rhenium (-325 mesh, 99.99\%, Alfa Aesar) and arsenic (-70 mesh, 99.99\%, Alfa Aesar) powders and indium ingots (99.95\%, Sigma Aldrich) were used as received. Phase purity of the starting materials was checked by using the standard X-ray diffraction technique, and in all cases no impurity phases were found. To synthesize the title solid solution, stoichiometric quantities of the starting elements were heated in evacuated silica tubes at $1073 \mathrm{~K}$ for 7 days with further cooling to room temperature in a shut off furnace. Firstly, the samples were analyzed by means of X-ray powder diffraction using a Stoe STADI-IP diffractometer with $\mathrm{Cu} \mathrm{K} \alpha_{1}$ radiation (Ge monochromator, $\lambda=1.540598 \AA$ ). To evaluate the lattice constants of the $\operatorname{Re}_{3} \mathrm{As}_{7-x} \mathrm{In}_{x}$ solid solution, all X-ray diffraction patterns were recorded with Ge as an internal standard ( $a=5.6576 \AA$ ). The data were treated with the program package Stoe WinXPOW. Secondly, the obtained samples were analyzed with a JSM JEOL scanning electron microscope operated at $20 \mathrm{kV}$ and equipped with an EDX detection system INCA x-Sight. Both point-spectra acquisition and element mapping were used to investigate the elemental and phase composition of the samples.

\section{Structure determination}

The crystal structure was determined by the Rietveld method from the X-ray powder diffraction data. For the sample with the nominal composition $\mathrm{Re}_{3} \mathrm{As}_{6.6} \mathrm{In}_{0.4}$, hereafter sample $\mathbf{S 1}$, the data were recorded with the Bruker D8 Advance diffractometer, $\mathrm{Cu} \mathrm{K} \alpha_{1}$ radiation (Ge monochromator, $\lambda=1.540598 \AA$ ). For the Rietveld refinements we used the TOPAS software (version 4.2, Bruker-AXS). The refinement enabled us to determine minor quantities of three impurity phases (Table 1) that were taken into account during the subsequent refinement. The atomic parameters taken from the crystal structure of $\operatorname{Re}_{3} \mathrm{As}_{7}$ [15] were

Table 1: Crystallographic data from the powder diffraction experiment for $\mathbf{S 1}$.

refined composition

formula weight $\left(\mathrm{g} \cdot \mathrm{mol}^{-1}\right)$

$T(\mathrm{~K})$

wavelength $(\AA)$

space group

cell dimensions, a $(\AA)$

$V\left(\AA^{3}\right)$

no. of formula units per cell

calculated density $\left(\mathrm{g} \cdot \mathrm{cm}^{-3}\right)$

$2 \theta$ range $\left({ }^{\circ}\right)$

$R_{\mathrm{p}}, R_{\mathrm{wp}}$, GOF

impurity phases (weight \%)
$\operatorname{Re}_{3} \mathrm{As}_{6.70(3)} \mathrm{In}_{0.30(3)}$

1095.041

300

1.540598

Im-3m (No. 229)

8.74231(6)

$668.157(14)$

4

10.88

17.00-85.01

$0.056,0.077,1.4$

Re $2.0 \%$, InAs $2.3 \%, \ln _{2} \mathrm{O}_{3} 1.0 \%$ 
Table 2: Atomic coordinates and displacement parameters for $\mathbf{S 1 .}$

\begin{tabular}{lllllll} 
site & Wyck. & $x$ & $y$ & $z$ & $B_{\text {iso }}\left(\AA^{2}\right)$ & occupancy \\
\hline $\operatorname{Re}$ & $12 \mathrm{e}$ & $0.3396(2)$ & 0 & 0 & $0.60(3)$ & $1 \mathrm{Re}$ \\
$\mathrm{E} 1$ & $12 \mathrm{~d}$ & $1 / 4$ & 0 & $1 / 2$ & $0.82(11)$ & $0.90(1) \mathrm{As}+0.10(1) \ln$ \\
As2 & $16 \mathrm{f}$ & $0.1662(2)$ & $0.1662(2)$ & $0.1662(2)$ & $0.82(5)$ & 1 As
\end{tabular}

used as the starting model. The refinement showed that the unique position of the rhenium atom was fully occupied. One of the two positions of the arsenic atoms, namely, the $12 \mathrm{~d}$ site, showed a remarkably low atomic displacement parameter and was subsequently refined as jointly occupied by indium and arsenic. The refinement led to the composition $\operatorname{Re}_{3} \mathrm{As}_{6.70(3)} \operatorname{In}_{0.30(3)}$ in reasonable agreement with the starting (synthetic) composition. Crystallographic details of the refinement are shown in Table 1, and the atomic parameters are shown in Table 2. Selected interatomic distances are listed in Table 3.

\begin{tabular}{ll} 
Table 3: Selected interatomic distances for $\mathbf{S 1}$ \\
bond & distance $(\AA)$ \\
\hline $\operatorname{Re}-\mathrm{E} 1 \times 4$ & $2.597(1)$ \\
$\operatorname{Re}-\mathrm{As} 2 \times 4$ & $2.553(1)$ \\
$\operatorname{Re}-\operatorname{Re} \times 1$ & $2.805(3)$ \\
As2-As2 $\times 1$ & $2.539(5)$ \\
As2-As2 $\times 3$ & $2.905(3)$
\end{tabular}

\section{Electronic-structure calculations}

The FPLO (full potential local orbitals) code was utilized for the electronic-structure calculations [16]. FPLO performs density functional calculations with the local density approximation (LDA) for the exchange-correlation potential [17]. The crystallographic data presented in Table 4 were used for the calculations [15]. The integrations in the $k$ space were performed by an improved tetrahedron method [18] on a grid of $16 \times 16 \times 16 k$ points evenly spread in the first Brillouin zone.

Table 4: $\operatorname{Re}_{3} \mathrm{As}_{7}$ crystallographic data used for electronic-structure calculations [15].

Space group $I m-3 m$ (No. 229), $a=8.7162(7) \AA$

\begin{tabular}{lllll}
\hline site & Wyck. & $x$ & $y$ & $z$ \\
\hline $\operatorname{Re}$ & $12 \mathrm{e}$ & $0.3406(9)$ & 0 & 0 \\
As1 & $12 \mathrm{~d}$ & $1 / 4$ & 0 & $1 / 2$ \\
As2 & $16 \mathrm{f}$ & $0.1687(20)$ & $0.1687(20)$ & $0.1687(20)$
\end{tabular}

\section{Physical property measurements}

For thermal transport measurements, the sample S1 was thoroughly ground and pressed at room temperature into a rectangular pellet of dimensions $8 \times 3 \times 2 \mathrm{~mm}^{3}$. The density of $\mathbf{S 1}$ was estimated from the linear sizes of the pellet to be about $70 \%$ of the theoretical density. This pellet was used to measure the electrical conductivity $(\sigma)$, the Seebeck coefficient $(S)$, and the thermal conductivity $(\kappa)$ in the temperature range of $77-300 \mathrm{~K}$ in a home-built setup. Resistance was determined from the voltage drops by applying a four-probe method in accordance with Ohm's law, i.e., $R=\Delta V / I$. The current $(I)$ was scanned in the range between $2.5 \mu \mathrm{A}$ and $16 \mathrm{~mA}$, and subsequently $\sigma$ was calculated after measuring the length between the contacts $(L)$ according to $\sigma=L /(A R)$, with the area $A=$ $3 \times 2 \mathrm{~mm}^{2}$. The Seebeck coefficient and thermal conductivity were measured by using an internal standard to determine the temperature difference in a custom-designed sample puck that was plugged into the cold finger of a closed-cycle refrigerator. All measurements were performed under dynamic vacuum.

For the magnetization measurements, powder samples of $\mathrm{Re}_{3} \mathrm{As}_{7}$ and $\mathbf{S 1}$ were loaded into plastic capsules. Measurements were performed with the VSM setup of Quantum Design PPMS in external fields of $0.1,0.5,1,2$, and $5 \mathrm{~T}$. To estimate the diamagnetic contribution from the sample holder, an empty capsule was measured under the same conditions.

\section{Results and Discussion Synthesis, sample characterization and crystal structure}

The synthesis of the $\mathrm{Re}_{3} \mathrm{As}_{7-x} \operatorname{In}_{x}$ series with $x=0,0.2,0.4,0.6$, 0.8 , and 1 from pure elements resulted in black powders that were stable in air. The obtained samples were analyzed by $\mathrm{X}$-ray powder diffraction. All samples showed reflections of the main phase of the $\mathrm{Re}_{3} \mathrm{As}_{7}$ type (space group $I m-3 m$ ), together with minor reflections of $\mathrm{Re}$, InAs, and $\mathrm{In}_{2} \mathrm{O}_{3}$ admixtures, the presence of which was also confirmed with EPMA (Figure 1). In order to obtain single-phase samples, we tried to improve the synthetic procedure, but neither increasing the annealing time nor pressing the reactants into pellets led to phase-pure samples. Some general trends should be noted. For the samples with $0 \leq x \leq 0.4$, absolute intensities and, thus, quantities of admixtures remain constant, while for $x>0.5$, quantities of Re and 


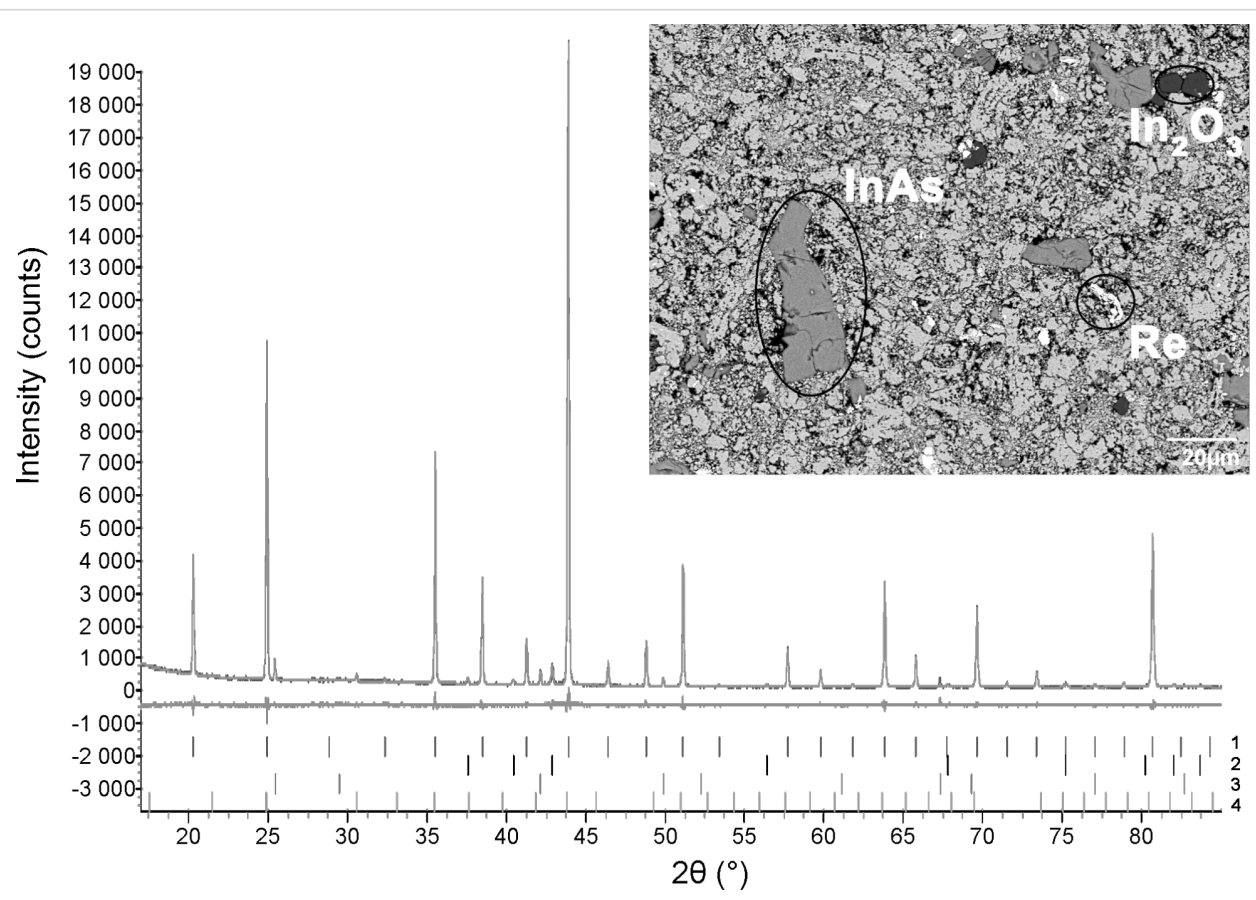

Figure 1: The plot of Rietveld refinement for the $\mathbf{S} 1$ sample. Experimental and difference curves, and positions of Bragg peaks are shown on the plot. Marked with numbers: 1: $\operatorname{Re}_{3} \mathrm{As}_{6.70(3)} \operatorname{In}_{0.30(3)} ; 2: \mathrm{Re} ; 3: \ln A s ; 4: \ln _{2} \mathrm{O}_{3}$. Inset: SEM micrograph of $\mathbf{S} 1$ showing the distribution of secondary phases in the microstructure (the most contaminated portion was chosen for showing all three admixtures).

InAs start to increase. Additionally, we found by a linear interpolation that the unit cell parameter of the $\mathrm{Re}_{3} \mathrm{As}_{7-x} \mathrm{In}_{x}$ solid solution increases up to $x=0.5$, and then remains constant at higher $x$ (Figure 2). All these facts suggest that the solid solution in question exists only for $x \leq 0.5$. The outermost composition $\mathrm{Re}_{3} \mathrm{As}_{6.5} \mathrm{In}_{0.5}$ possesses exactly 55 valence electrons per formula unit. As mentioned above, this electron concentration should yield the semiconducting behavior for compounds with

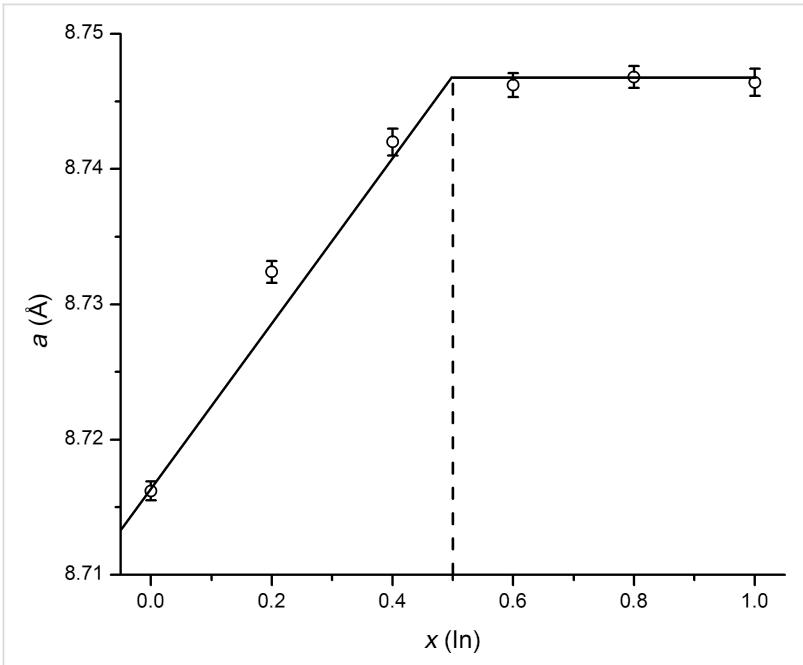

Figure 2: Dependence of the $\operatorname{Re}_{3} A s_{7-x} \mathrm{In}_{x}$ cubic-unit-cell parameter on the nominal indium content. Esd's are calculated from least-squares fits of the powder data. the $\mathrm{Ir}_{3} \mathrm{Ge}_{7}$ structure type. Thus, the indium substitution for arsenic in $\operatorname{Re}_{3} \mathrm{As}_{7}$ could be used as a chemical modification to control transport properties of this system.

The crystal structure of the solid solution was studied for the $\mathbf{S 1}$ sample by the Rietveld method from X-ray powder diffraction data (Figure 1, Table 1 and Table 2). The title compound crystallizes with the $\operatorname{Ir}_{3} \mathrm{Ge}_{7}$ crystal structure (Figure 3). This struc-

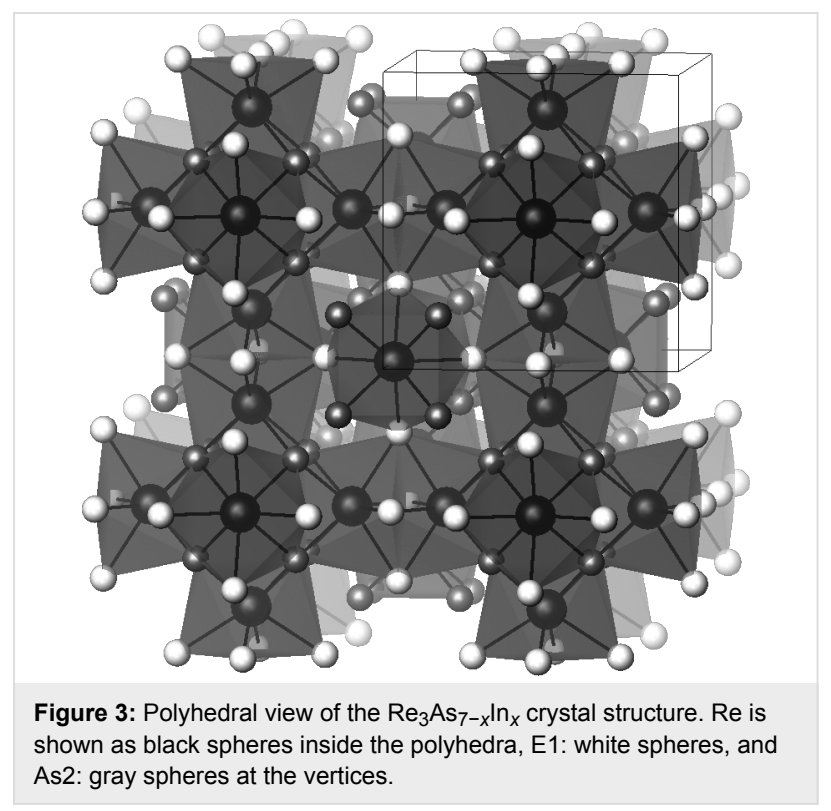


ture can be described as being composed of rhenium-centered square antiprisms of $\mathrm{E}$ atoms, $\mathrm{ReE}_{8}(\mathrm{E}=\mathrm{As} / \mathrm{In})$. Two square antiprisms are linked by sharing a square face. These pairs form the so-called $\operatorname{Re}_{2} \mathrm{E}_{12}$ barrels, the main building blocks of the crystal structure. The barrels, oriented along the main crystallographic directions, form two interpenetrating 3D networks in accordance with the body-centering and, thus, build up the entire crystal structure.

The formation of the solid solution may be associated with a chemical substitution on different crystallographic sites. There are two sites forming the coordination polyhedra of $\mathrm{E}$ atoms in the $\operatorname{Ir}_{3} \mathrm{Ge}_{7}$ structure type (Figure 4), and the substitution is possible for both sites depending on the chemical nature of the E elements. It is known from the literature that in the case of the Ge for As substitution in the parent compound $\mathrm{Re}_{3} \mathrm{As}_{7}$, all $\mathrm{Ge}$ atoms enter the As2 (16f) site [13]. In contrast, we have found that when indium substitutes for arsenic in $\mathrm{Re}_{3} \mathrm{As}_{7}$, all indium atoms are on the E1 (12d) site. The preference for the certain As position depends on different aspects, including size, nuclear charge, and number of valence electrons of the heteroatom. In particular, there is an E-E single bond between atoms occupying the $16 \mathrm{f}$ site, with a bond distance of 2.538(5) A. Clearly, indium does not favor such a short bond to arsenic and, therefore, avoids the occupation of this site.
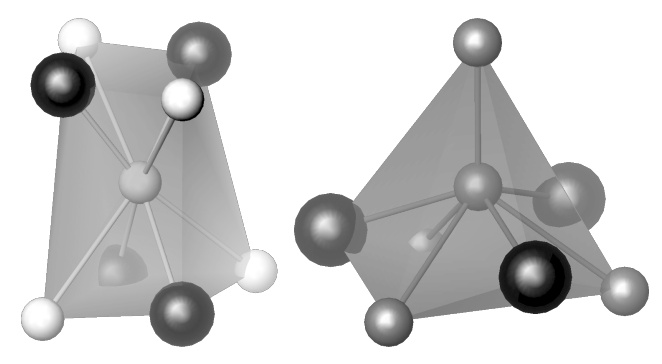

Figure 4: Coordination polyhedra of E1 (left) and As2 (right) sites in the crystal structure of $\mathrm{Re}_{3} A s_{7-x} \mathrm{In}_{x}$.

\section{Electronic structure, magnetic and thermo- electric properties}

The computed density of states for $\mathrm{Re}_{3} \mathrm{As}_{7}$ is shown in Figure 5. The Fermi level lies slightly above the gap of $0.8 \mathrm{eV}$ that separates the conduction band from the valence band. The nonzero DOS at $E_{\mathrm{F}}$ implies metallic behavior for the undoped $\mathrm{Re}_{3} \mathrm{As}_{7}$. Additionally, the steep slope of the DOS curve near $E_{\mathrm{F}}$ should lead to a high Seebeck coefficient according to $S \sim 1 / N \cdot \partial N\left(E_{\mathrm{F}}\right) / \partial E[19]$, provided that the system is made semiconducting by doping. Indeed, the absolute values of $S$ for $\mathrm{Re}_{3} \mathrm{As}_{6.4} \mathrm{Ge}_{0.6}$ exceed $150 \mu \mathrm{V} \cdot \mathrm{K}^{-1}$ at high temperatures, thus leading to high values of $Z T$ [13].

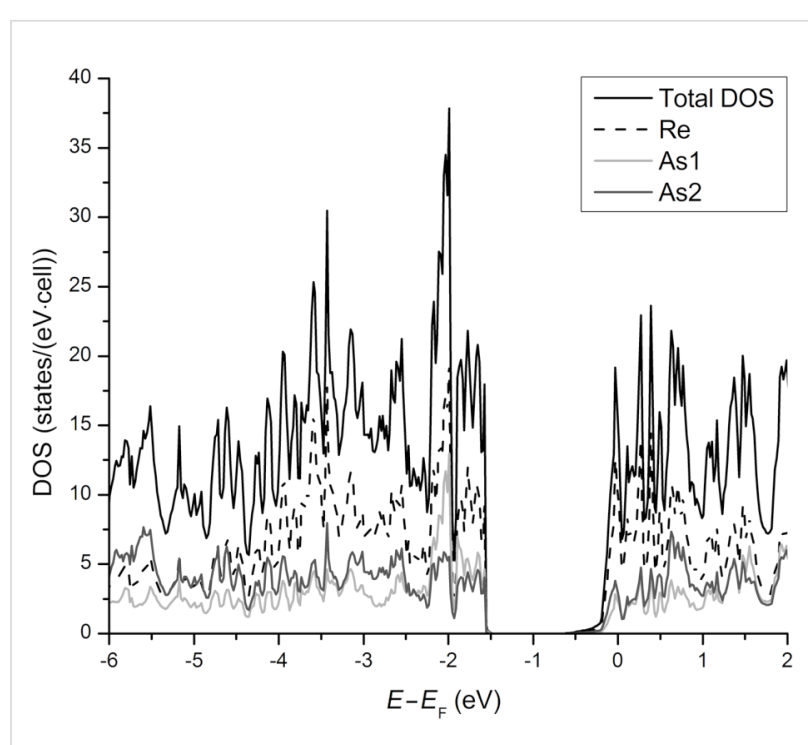

Figure 5: Density-of-states curve for $\mathrm{Re}_{3} A s_{7}$. Re contribution: dashed line, As1 and As2: light and dark gray lines, respectively.

In $\mathrm{Re}_{3} \mathrm{As}_{7}$, the calculated density of states at $E=E_{\mathrm{F}}$ is 8.3 states/(eV·f.u.). For the solid solution $\operatorname{Re}_{3} \mathrm{As}_{6.7} \mathrm{In}_{0.3}$ (the composition obtained from the Rietveld refinement of the X-ray powder diffraction data, see Table 1), the DOS is reduced to 5.15 states/(eV·f.u.), given the rigid-band shift with the assumption that $\mathrm{Re}_{3} \mathrm{As}_{7}$ possesses 56 valence electrons per f.u. and $\operatorname{Re}_{3} \mathrm{As}_{6.7} \operatorname{In}_{0.3} 55.4$ electrons. Therefore, both compounds should be metallic with a Pauli paramagnetic contribution to the total susceptibility $\chi=\chi_{\text {dia }}+\chi_{\mathrm{P}}$, where $\chi_{\text {dia }}$ is core diamagnetism, and $\chi_{\mathrm{P}}=\mu_{\mathrm{B}}{ }^{2} \cdot N\left(E_{\mathrm{F}}\right)$, with $\mu_{\mathrm{B}}$ being the Bohr magneton [20]. The formula yields $\chi_{\mathrm{P}}=9 \times 10^{-5}$ and $5.5 \times 10^{-5} \mathrm{emu} / \mathrm{mol}$ for $\mathrm{Re}_{3} \mathrm{As}_{7}$ and $\mathbf{S 1}$, respectively.

Experimentally, both $\mathrm{Re}_{3} \mathrm{As}_{7}$ and $\mathbf{S} 1$ show substantial diamagnetism in the examined temperature range. However, the susceptibility curves, Figure 6, lie above the level of core diamagnetism $\chi_{\text {dia }}=-3.37 \times 10^{-4} \mathrm{emu} / \mathrm{mol}$, computed for a combination of $\mathrm{Re}^{7+}$ and $\mathrm{As}(\mathrm{V})$ [21]. Therefore, both pure and In-doped $\mathrm{Re}_{3} \mathrm{As}_{7}$ feature an additional paramagnetic contribution to the susceptibility. The experimental value of $\chi_{\mathrm{P}}=$ $\chi-\chi_{\text {dia }}=1.20(7) \times 10^{-4} \mathrm{emu} / \mathrm{mol}$ for $\mathrm{Re}_{3} \mathrm{As}_{7}$ is reasonably close to the one expected from the DOS at $E_{\mathrm{F}}$. However, the calculation of $\chi_{\mathrm{P}}$ substantially depends on the estimation method of $\chi_{\text {dia }}$, especially for a compound that cannot be considered as ionic. For this reason, the calculated value of $\chi_{P}$ may differ from the experimental one. The susceptibility of the S1 slightly decreases upon cooling but starts increasing below $80 \mathrm{~K}$. While the low-temperature upturn could be due to a small number of paramagnetic impurities, the conspicuous increase in $\chi$ above $80 \mathrm{~K}$ does not conform to the Pauli paramagnetism and reflects deviations of $\mathbf{S 1}$ from a simple metal. 


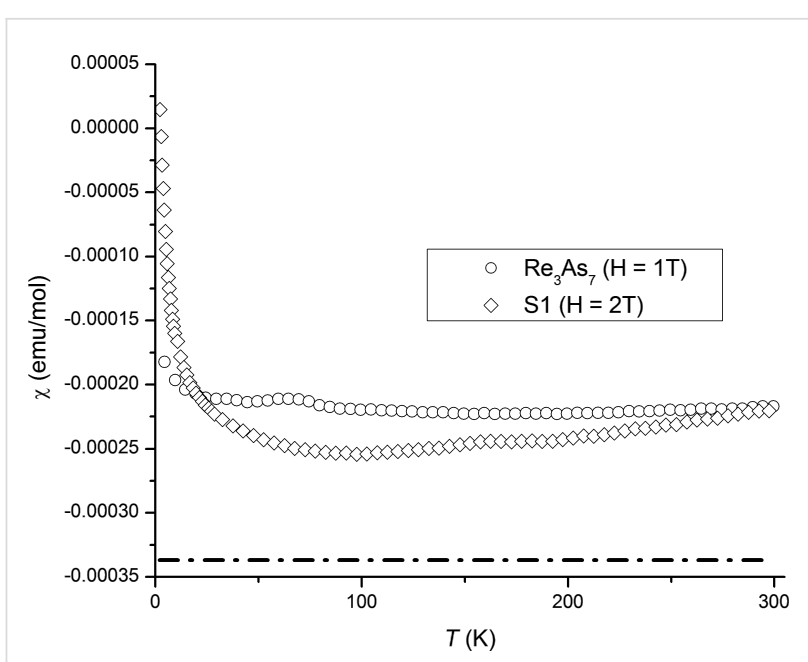

Figure 6: Magnetic susceptibility-versus-temperature plots for the $\mathrm{Re}_{3} \mathrm{As}_{7}$ and $\mathbf{S} 1$ samples. The contribution of core diamagnetism is shown as a dash-dotted line.

Figure 7 compiles the plots of the electric conductivity $(\sigma)$, Seebeck coefficient $(S)$, thermal conductivity $(\kappa)$, and $Z T$ in the temperature range of $77-300 \mathrm{~K}$ for S1. $S$ is negative in this temperature range, evidencing that the $\mathbf{S} 1$ is an n-type conductor. However, the $\sigma$-versus- $T$ behavior for the $\mathbf{S 1}$ sample is neither metallic nor classically semiconducting, because $\sigma$ increases almost linearly with temperature. Thus, $\mathbf{S 1}$ can be regarded as a bad metal or degenerate semiconductor, consid- ering the possible presence of defects, such as vacancies in its crystal structure, which was proposed earlier for $\mathrm{Re}_{3} \mathrm{As}_{7}$ [15]. In the $\mathrm{Ir}_{3} \mathrm{Ge}_{7}$ family, $\mathrm{Re}_{3} \mathrm{As}_{7-x} \mathrm{Ge}_{x}$ exhibits n-type conductivity [13], while $\mathrm{Mo}_{3} \mathrm{Sb}_{5+\delta} \mathrm{Te}_{2-\delta}$ is a p-type conductor [11]. The obtained values of $S$ for $\mathbf{S} \mathbf{1}$ are comparable with those for $\mathrm{Re}_{3} \mathrm{As}_{6.4} \mathrm{Ge}_{0.6}$ and $\mathrm{Mo}_{3} \mathrm{Sb}_{5.4} \mathrm{Te}_{1.6}:-49,-72$, and $+55 \mu \mathrm{V} \cdot \mathrm{K}^{-1}$ at $300 \mathrm{~K}$, respectively $[11,13]$. Moreover, the extent of the substitution $x$ in the $\mathrm{Re}_{3} \mathrm{As}_{7-x} \mathrm{In}_{x}$ solid solution can be further optimized, and possibly lead to larger values of $S$. Unfortunately, the $\mathbf{S 1}$ displays considerably lower values of the electrical conductivity compared to both $\mathrm{Re}_{3} \mathrm{As}_{7-x} \mathrm{Ge}_{x}$ and $\mathrm{Re}_{3} \mathrm{As}_{7-x} \mathrm{Sn}_{x}(0.1 \leq x \leq 0.6)$ [22]. For instance, the roomtemperature value of $1090 \Omega^{-1} \cdot \mathrm{cm}^{-1}$ for $\mathrm{Re}_{3} \mathrm{As}_{6.4} \mathrm{Ge}_{0.6}$ [13] is about 3000 times larger than the observed value of $3.8 \Omega^{-1} \cdot \mathrm{cm}^{-1}$ for $\mathbf{S 1}$.

The thermal conductivity of the sample $\mathbf{S 1}$ is quite low. Its room-temperature value is $0.3 \mathrm{~W} \cdot \mathrm{m}^{-1} \cdot \mathrm{K}^{-1}$, which is an order of magnitude lower than for the Ge- and Sn-substituted compounds. This may be caused by two factors: Firstly, it could be attributed to the preference of indium atoms for only one position within the crystal structure (increased structural complexity); secondly, relatively low density of the sample (about $70 \%$ ) may diminish the thermal conductivity due to the sample porosity. The total thermal conductivity is a sum of the electronic $\left(\kappa_{\mathrm{e}}\right)$ and lattice $\left(\kappa_{\mathrm{L}}\right)$ parts. Taking into account the rather low electrical conductivity and applying the
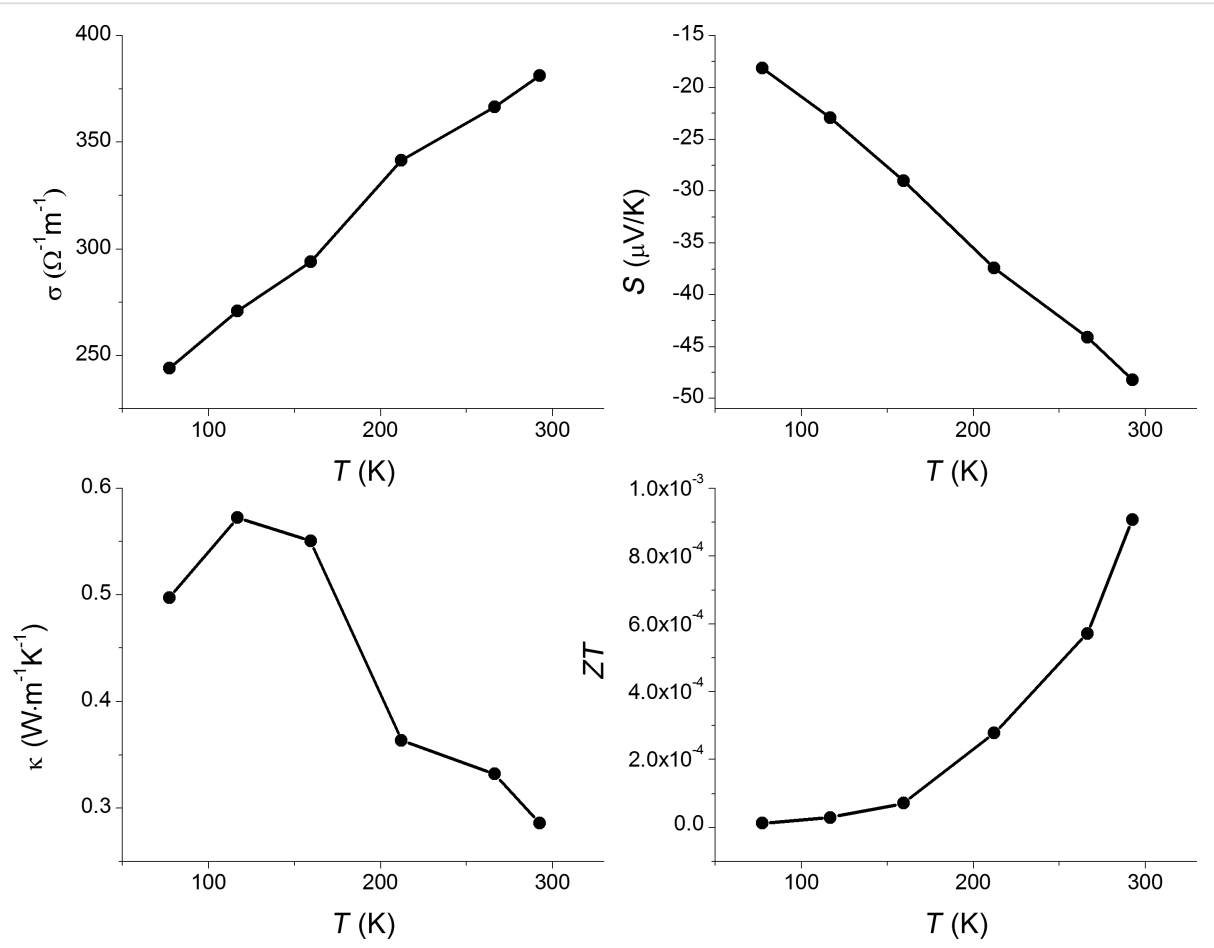

Figure 7: Thermoelectric properties of the $\mathbf{S} 1$ sample as a function of temperature. Solid lines are drawn to guide the eye. 
Wiedemann-Franz relation $\kappa_{\mathrm{e}}=\sigma L T$, where $L$ is the ideal Lorentz number, we estimate that the electronic part of the total thermal conductivity is negligibly small, and the observed value is essentially the lattice contribution to the thermal conductivity.

Combining the electrical conductivity, Seebeck coefficient and thermal conductivity, we calculate the temperature dependence of $Z T$ shown in Figure 7. $Z T$ increases with temperature, and reaches $Z T=0.0008$ at room temperature, which is 30 times lower than for $\mathrm{Re}_{3} \mathrm{As}_{7-x} \mathrm{Ge}_{x}$ [13]. Given the compositional width of the $\operatorname{Re}_{3} \mathrm{As}_{7-x} \mathrm{In}_{x}$ solid solution and the low thermal conductivity of the investigated sample, we note that the optimum combination of $S$ and $\sigma$ for $\operatorname{Re}_{3} \mathrm{As}_{7-x} \mathrm{In}_{x}$ is still to be found.

\section{Conclusion}

Chemical modification of $\operatorname{Re}_{3} \mathrm{As}_{7}$ resulted in the formation of the new $\mathrm{Re}_{3} \mathrm{As}_{7}$-based solid solution $\mathrm{Re}_{3} \mathrm{As}_{7-x} \mathrm{In}_{x}(x \leq 0.5)$ with an $\mathrm{Ir}_{3} \mathrm{Ge}_{7}$ type of crystal structure. The indium for arsenic substitution occurs exclusively on the $12 \mathrm{~d}$ site, thus keeping intact the As-As dumbbells with $d_{\mathrm{As}-\mathrm{As}}=2.538(5) \AA$. While $\mathrm{Re}_{3} \mathrm{As}_{7}$ shows a Pauli paramagnetic contribution to the magnetic susceptibility in line with the results of band-structure calculations, the $\mathbf{S 1}$ sample behaves as a bad metal or heavily doped semiconductor, with electrons being the dominant charge carriers. This compound combines low thermal conductivity with a relatively low electrical conductivity, and therefore, its thermoelectric figure of merit $Z T$ reaches only 0.0008 at room temperature. Further optimization of the thermoelectric properties by varying the chemical composition of $\mathrm{Re}_{3} \mathrm{As}_{7-x} \mathrm{In}_{x}$ is proposed.

\section{Acknowledgements}

The work is supported in part by the Mobilitas grant of the ESF, the Lomonosov Moscow State University Program of Development, and the Russian Foundation for Basic Research.

\section{References}

1. Shevelkov, A. V. Russ. Chem. Rev. 2008, 77, 3-21. doi:10.1070/RC2008v077n01ABEH003746

2. Rowe, D. M. CRC Handbook of Thermoelectrics; CRC Press: Boca Raton, FL, 1995. doi:10.1201/9781420049718

3. Heremans, J. P.; Jovovic, V.; Toberer, E. S.; Saramat, A.; Kurosaki, K.; Charoenphakdee, A.; Yamanaka, S.; Snyder, G. J. Science 2008, 321, 554-557. doi:10.1126/science.1159725

4. Venkatasubramanian, R.; Colpitts, T.; Watko, E.; Lamvik, M.; El-Masry, N. J. Cryst. Growth 1997, 170, 817-821. doi:10.1016/S0022-0248(96)00656-2

5. Shi, X.; Yang, J.; Salvador, J.; Chi, M.; Cho, J.; Wang, H.; Bai, S.; Yang, J.; Zhang, W.; Chen, L. J. Am. Chem. Soc. 2011, 133, 7837-7846. doi:10.1021/ja111199y

6. Rogl, G.; Grytsiv, A.; Rogl, P.; Bauer, E.; Zehetbauer, M. Intermetallics 2011, 19, 546-555. doi:10.1016/j.intermet.2010.12.001
7. Shevelkov, A. V.; Kovnir, K. Zintl Clathrates. In Zintl Phases; Fässler, T. F., Ed.; Structure and Bonding, Vol. 139; Springer-Verlag: Berlin Heidelberg, 2011; pp 97-142. doi:10.1007/430_2010_25

8. Iverson, B. B. J. Mater. Chem. 2010, 20, 10778-10787. doi:10.1039/c0jm02000a

9. Zaikina, J. V.; Mori, T.; Kovnir, K. A.; Teschner, D.; Senyshin, A.; Schwarz, U.; Grin, Y.; Shevelkov, A. V. Chem.-Eur. J. 2010, 16, 12582-12589. doi:10.1002/chem.201001990

10. Gougeon, P.; Gall, P.; Al Orabi, R. A. R.; Fontaine, B.; Gautier, R.; Potel, M.; Zhou, T.; Lenoir, B.; Colin, M.; Candolfi, C.; Dauscher, A. Chem. Mater. 2012, 24, 2899-2908. doi:10.1021/cm3009557

11. Gascoin, F.; Rasmussen, J.; Snyder, G. J. J. Alloys Compd. 2007, 427, 324-329. doi:10.1016/j.jallcom.2006.03.030

12. Soheilnia, N.; Giraldi, J.; Assoud, A.; Zhang, H.; Tritt, T. M.; Kleinke, H. J. Alloys Compd. 2008, 448, 148-152. doi:10.1016/j.jallcom.2006.10.056

13. Soheilnia, N.; Xu, H.; Zhang, H.; Tritt, T. M.; Swainson, I.; Kleinke, H. Chem. Mater. 2007, 19, 4063-4068. doi:10.1021/cm0708517

14. Häussermann, U.; Elding-Pontén, M.; Svensson, C.; Lidin, S. Chem.-Eur. J. 1998, 4, 1007-1015. doi:10.1002/(SICI)1521-3765(19980615)4:6<1007::AID-CHEM1007>3. 3.CO;2-Z

15. Jensen, P.; Kjekshus, A.; Skansen, T. J. Less-Common Met. 1969, 17, 455-458. doi:10.1016/0022-5088(69)90073-3

16. Koepernik, K.; Eschrig, H. Phys. Rev. B 1999, 59, 1743-1757. doi:10.1103/PhysRevB.59.1743

17. Perdew, J. P.; Wang, Y. Phys. Rev. B 1992, 45, 13244-13249. doi:10.1103/PhysRevB.45.13244

18. Blöchl, P. E.; Jepsen, O.; Andersen, O. K. Phys. Rev. B 1994, 49, 16223-16233. doi:10.1103/PhysRevB.49.16223

19. Kleinke, H. Chem. Mater. 2010, 22, 604-611. doi:10.1021/cm901591d

20. Ashcroft, N. W.; Mermin, N. D. CRC Solid State Physics; Brooks/Cole: Belmont, CA, 1976.

21. Bain, G. A.; Berry, J. F. J. Chem. Educ. 2008, 85, 532-536. doi:10.1021/ed085p532

22. Xu, H.; Holgate, T.; He, J.; Su, Z.; Tritt, T. M.; Kleinke, H. J. Electron. Mater. 2009, 38, 1030-1036. doi:10.1007/s11664-008-0623-0

\section{License and Terms}

This is an Open Access article under the terms of the Creative Commons Attribution License (http://creativecommons.org/licenses/by/2.0), which permits unrestricted use, distribution, and reproduction in any medium, provided the original work is properly cited.

The license is subject to the Beilstein Journal of Nanotechnology terms and conditions: (http://www.beilstein-journals.org/bjnano)

The definitive version of this article is the electronic one which can be found at:

$\underline{\text { doi:10.3762/bjnano. } 4.52}$ 